\title{
Técnica de Restrição Hídrica: Efeito sobre Acremonium strictum, Protrusão de Sementes e Obtenção de Sementes de Milho Infetadas*
}

\author{
Hudson Teixeira ${ }^{1}$, José da Cruz Machado ${ }^{2}$, Denis Oride² ${ }^{2}$ Marcelo C. Alves ${ }^{2} \&$ Alexandre Noda ${ }^{2}$ \\ ${ }^{1}$ Departamento de Fitopatologia, Universidade Federal de Viçosa, CEP 36570-000, Viçosa, MG, tel. (31) 3899-1096, \\ e-mail: hudsont@ufv.br; ${ }^{2}$ Departamento de Fitopatologia, Universidade Federal de Lavras, Cx. Postal 37, CEP 37200-000, \\ Lavras, MG, tel. (35) 3829-1470, e-mail: machado@ufla.br
}

(Aceito para publicação em 14/12/2004)

Autor para correspondência: Hudson Teixeira

TEIXEIRA, H., MACHADO J.C., ORIDE, D., ALVES, M.C. \& NODA, A. Técnica de restrição hídrica: efeito sobre Acremonium strictum, protrusão de sementes e obtenção de sementes de milho infetadas. Fitopatologia Brasileira 30:109-114. 2005.

\section{RESUMO}

Este experimento objetivou a obtenção de sementes de milho (Zea mays) portadoras de Acremonium strictum, utilizandose técnica de restrição hídrica e visando sua utilização posterior em estudos sobre a interação patógeno - hospedeiro. O trabalho constou de avaliações de efeitos da adição de manitol, $\mathrm{NaCl}$ e $\mathrm{KCl}$ nos potenciais de - 0,$48 ;-0,6 ;-0,8 ;-1,0 ;-1,2$ e -1,4 $\mathrm{MPa}$ ao meio extrato de malte-ágar (MEA) sobre o crescimento micelial, textura de micélio, forma marginal da colônia, pigmentação e esporulação in vitro de colônias de $A$. strictum, bem como sobre o percentual de protrusão de radículas de sementes de milho. Foi avaliada também a correlação entre diferentes períodos de exposição das sementes à colônia do fungo e sua incidência em sementes desinfestadas ou não com $\mathrm{NaClO} 1 \%$ por $2 \mathrm{~min}$. A taxa de crescimento micelial de A. strictum foi estimulada em meio MEA + manitol (-0,6 a -1,4 MPa) e a morfologia típica de colônias do patógeno em meio MEA acrescido com manitol, $\mathrm{NaCl}$ e $\mathrm{KCl}$ diferiu dos padrões apresentados no controle (-0,48 MPa). A esporulação do fungo foi estimulada pelo manitol nos potenciais $-0,8$ a $-1,4 \mathrm{MPa}$, aplicados ao meio de cultivo padrão. As menores percentagens de protrusão radicular em sementes de milho incubadas por até cinco dias foram observadas em MEA + manitol (-1,4 MPa) e MEA + $\mathrm{NaCl}(-1,2 \mathrm{e}$ -1,4 MPa). No entanto, verificou-se efeito fitotóxico do $\mathrm{NaCl}$ sobre a germinação das sementes. Por meio da técnica de restrição hídrica foi possível obter até $36 \%$ de sementes de milho infetadas por $A$. strictum.

Palavras-chave adicionais: fungo, sementes, inoculação, infecção, protrusão radicular.

\begin{abstract}
Water restriction technique: effect on Acremonium strictum, seeds protusion and obtaining of maize seeds infected

This work was carried out to obtain maize (Zea mays) seeds with Acremonium strictum, by means of an adapted water restriction technique, in order to use them in pathogen-host studies. An evaluation was made of the effects of mannitol, $\mathrm{NaCl}$ and $\mathrm{KCl}$ at potentials of $-0.48 ;-0.6 ;-0.8 ;-1.0 ;-1.2$ and $-1.4 \mathrm{MPa}$ in malt extract agar medium (MEA) on the colony morphology and sporulation of $A$. strictum, and on radicle protrusion of maize seeds. Also evaluated was the correlation between different exposition periods of the seeds to the fungal colony and its incidence on disinfested and non-disinfested seeds with $\mathrm{NaClO} 1 \%$ ( $2 \mathrm{~min}$ ). The micelial growth of $A$. strictum was stimulated in MEA + mannitol medium at potentials of -0.6 to $-1.4 \mathrm{MPa}$. The morphology of $A$. strictum colonies in MEA medium for all the solutes used differed from the colonies in the control treatment $(-0.48 \mathrm{MPa})$. Fungal sporulation was stimulated by mannitol on potentials -0.8 to $-1.4 \mathrm{MPa}$ in MEA. The lowest percentages of radicle protrusion in maize seeds were observed in cases when seeds were exposed to water restriction induced by MEA + mannitol at potential of $-1,4 \mathrm{MPa}$; $\mathrm{MEA}+\mathrm{NaCl}$ at $-1,2$ and $-1,4 \mathrm{MPa}$. The $\mathrm{NaCl}$, however, was verified to cause toxic effects in seed germination. This work shows that it is possible to obtain A. strictum infection in $36 \%$ of maize seeds infected by means of water restriction.
\end{abstract}

Additional keywords: fungus, seed, inoculation, infection, protrusion in maize seeds.

\section{INTRODUÇÃO}

Patógenos causadores de podridão de sementes, morte de plântulas e podridão de colmos são considerados importantes na cultura do milho (Zea mays L.), onde podem

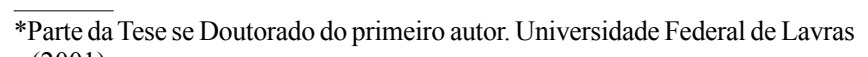
(2001). interferir diretamente no estande, vigor e produtividade final das plantas. Dentre os principais agentes causais desses tipos de enfermidades na cultura do milho, destaca-se o fungo Acremonium strictum W. Gams (sin.: Cephalosporium acremonium Auct. non Corda) (Richardson, 1980; Smith \& White, 1988; Shurtleff, 1992). Também a interação deste com seu hospedeiro tem sido pouco estudada. 
A inoculação de fungos em sementes é uma prática útil em patologia de sementes para a condução de experimentos e entendimento de certos aspectos que envolvam a interação patógeno-hospedeiro. Dentre os métodos convencionais de inoculação de sementes são citados: imersão em suspensão de conídios (Tanaka, 1990); infiltração forçada do inóculo fúngico por meio de vácuo (Rava \& Sartorato, 1996; Chaves et al., 1999); mistura de formulação em pó de caulim e esporos do fungo (Vieira, 1996; Teixeira et al., 1997a); mistura de massa micelial e esporos (Tanaka \& Corrêa, 1981); e exposição de sementes à colônia fúngica por períodos limitados de tempo (Peres, 1996; Teixeira et al., 1997b). Grande parte dos métodos citados, disponibiliza umidade suficiente para inicializar o processo de embebição e germinação das sementes. Este fenômeno normalmente promove modificações físicas e/ou fisiológicas indesejáveis, além de não assegurar a infecção pelo patógeno em níveis desejados.

A metodologia de condicionamento osmótico ou restrição hídrica (Heydecker et al., 1973; Heydecker et al., 1975), originalmente desenvolvida visando à melhoria da qualidade fisiológica de sementes, foi adaptada na Universidade Federal de Lavras em estudos envolvendo ensaios de inoculação de sementes com patógenos. Nesse método, o potencial hídrico do meio agarizado, sobre o qual desenvolver-se-á a colônia do patógeno, é controlado pela adição de solutos, por exemplo, polietileno glicol (PEG), manitol, $\mathrm{KCl}, \mathrm{NaCl}, \mathrm{NaOH}, \mathrm{MgSO}_{4}, \mathrm{MgCl}_{2}, \mathrm{~K}_{3} \mathrm{PO}_{4}$, $\mathrm{KH}_{2} \mathrm{PO}_{4}$, glicerol, sacarose (Pill, 1994). Nesse processo, a germinação das sementes pode ser inibida ou retardada, o que possibilita a exposição destas à colônia do patógeno por períodos mais prolongados e variáveis, obtendo-se como conseqüência, maiores percentuais de infecção. Nesta linha, Carvalho (1999) conseguiu a inoculação de sementes de feijoeiro (Phaseolus vulgaris L.) com Colletotrichum lindemuthianum (Sacc. \& Magn.) Bri. \& Cav., em meio batatadextrose-ágar (BDA) ajustado osmoticamente com manitol e PEG 6000. Também verificou que esta técnica aumentou a eficiência do processo de inoculação de sementes. Costa (2000), utilizando o meio batata-sacarose-ágar (BSA) acrescido de $\mathrm{KCl}$, inoculou sementes de feijoeiro com Fusarium oxysporum Schlecht. f.sp. phaseoli Kendrick \& Snyder e obteve de 64\% a $70 \%$ de incidência média do patógeno.

Visando obter mais informações e melhoria nas técnicas de inoculação de sementes, este trabalho objetivou adaptar a técnica de restrição hídrica para obtenção de sementes de milho infetadas por $A$. strictum, em condições controladas e sem interferência na qualidade fisiológica das mesmas.

\section{MATERIAL E MÉTODOS}

\section{Obtenção e multiplicação do inóculo de $\boldsymbol{A}$. strictum}

$\mathrm{O}$ isolado de $A$. strictum empregado foi obtido aleatoriamente de sementes de milho, submetidas ao teste de sanidade pelo método de incubação em papel de filtro (Limonard, 1966). O fungo foi isolado para meio extrato de malte-ágar (MEA) (Smith \& Onions, 1994) e mantido em câmara de incubação do tipo BOD, na temperatura constante de $20^{\circ} \mathrm{C}$, fotoperíodo de $12 \mathrm{~h}$ de luz, por dez dias.

\section{Efeito da restrição hídrica do meio MEA no desenvolvimento de colônias de $\boldsymbol{A}$. strictum}

O meio MEA foi ajustado osmoticamente para $-0,48$ (controle); -0,6; -0,8; -1,0; -1,2 e -1,4 MPa (Megapascal) através da adição de manitol $\left(\mathrm{C}_{6} \mathrm{H}_{14} \mathrm{O}_{6}\right)$, cloreto de potássio $(\mathrm{KCl})$ e cloreto de sódio $(\mathrm{NaCl})$, em separado. As doses dos solutos, adicionadas ao meio, buscando atingir os níveis de restrição hídrica desejados foram calculadas empregando-se o software SPPM (Michel \& Radcliffe, 1995), tendo como base potencial osmótico do meio MEA igual a -0,48 $\mathrm{MPa}$ (Sommers et al., 1970). As concentrações dos solutos utilizados para obtenção dos diversos ajustes osmóticos do meio padrão MEA, em g/l, estão descritas na Tabela 1.

Para o centro de cada placa de Petri de vidro de $9 \mathrm{~cm}$ de diâmetro, contendo $20 \mathrm{ml}$ de meio MEA com os diferentes potenciais hídricos de cada soluto, foi transferido um disco de $5 \mathrm{~mm}$ de diâmetro, retirado de colônias contendo micélio de $A$. strictum e incubado em BOD, a $20{ }^{\circ} \mathrm{C}$, fotoperíodo de $12 \mathrm{~h}$ de luz, por dez dias. $\mathrm{O}$ diâmetro médio das colônias desenvolvidas foi mensurado a cada dois dias. A taxa de crescimento micelial foi determinada pelo somatório dos quocientes entre o diâmetro médio da colônia do patógeno e o número de dias decorridos até a avaliação. Também foi determinada a influência da restrição hídrica sobre algumas características morfológicas das colônias de A. strictum, como crescimento micelial típico (superficial - $\mathrm{S}$ ou aéreo - A), textura do micélio (lisa - L, floculosa - F ou cotonosa - C), forma marginal da colônia (regular - R ou irregular - I) e pigmentação do anverso e verso da colônia. Para a avaliação desta característica utilizou-se como referência a carta de cores de Munsell (1976): bege - B (2.5Y - 8.5/2); rosa claro - Rc (2.5R - 9/2); rosa salmão - Rs (10R - 7/10) ou rosa alaranjado - Ra $(10 \mathrm{R}-6 / 14)$.

A esporulação de colônias de $A$. strictum foi determinada em meio MEA nos potenciais hídricos - 0,48 (controle); -0,8; 1,$0 ;-1,2$ e $-1,4 \mathrm{MPa}$ de manitol e $\mathrm{NaCl}$, aos dez dias de incubação. A partir de cada colônia pura obtida foram preparadas suspensões de esporos utilizando-se $5 \mathrm{ml}$ de água destilada esterilizada, aos quais adicionou-se $0,1 \%$ de Tween 80. Em seguida, efetuou-se a raspagem superficial da colônia fúngica com um pincel fino esterilizado e a suspensão de esporos obtida foi filtrada em gaze estéril. A esporulação foi determinada utilizando-se câmara de Neubauer e os resultados foram expressos em número de esporos por mililitro de suspensão fúngica.

\section{Efeito do ajuste osmótico do meio MEA na protrusão radicular de sementes de milho}

Foram empregadas sementes de milho da cultivar C805 (Peneira R1, safra 1999/2000, produzida na região de Nova Fátima-PR) com 95\% de germinação. As sementes foram desinfestadas com $\mathrm{NaClO} 1 \%$ por 2 min, enxaguadas em água destilada por duas vezes sucessivas e secas em câmara de 
Técnica de restrição hídrica: efeito sobre Acremonium strictum, protrusão...

fluxo laminar por $2 \mathrm{~h}$. Para cada placa de Petri de $15 \mathrm{~cm}$ de diâmetro contendo $40 \mathrm{ml}$ de meio MEA foram distribuídas 25 sementes. As concentrações dos solutos utilizados foram ajustadas em - 0,48 (controle); $-0,8 ;-1,0 ;-1,2 \mathrm{e}-1,4 \mathrm{MPa}$ por meio da adição de manitol e $\mathrm{NaCl}$, em separado (Tabela 1 ). $\mathrm{O}$ soluto $\mathrm{KCl}$ (cloreto de potássio) não foi utilizado, pois no ensaio anterior apresentou-se inferior aos demais solutos avaliados quanto ao crescimento micelial de $A$. strictum (Tabela 2). As sementes em meio agarizado MEA foram incubadas em BOD, a $20^{\circ} \mathrm{C}$, fotoperíodo de $12 \mathrm{~h}$, durante cinco e oito dias, quando foi determinado o percentual de sementes com protrusão radicular superior a $1 \mathrm{~mm}$.

Nível de infecção de sementes de milho incubadas sobre colônias de $A$. strictum desenvolvidas em meio MEA osmoticamente modificado

Objetivando obter colônias puras, inóculo de $A$. strictum foi cultivado por dez dias em BOD, a $20^{\circ} \mathrm{C}$ e fotoperíodo de $12 \mathrm{~h}$. Em seguida, o mesmo foi multiplicado em placas de Petri de vidro, de $9 \mathrm{~cm}$ de diâmetro, esterilizadas, contendo $20 \mathrm{ml}$ de meio MEA. Sob condições assépticas, 5,0 $\mathrm{ml}$ de água destilada esterilizada e $0,1 \%$ de Tween 80 foram depositados em cada placa. Através da raspagem superficial das colônias, utilizando um pincel fino, foi obtida a suspensão de esporos, cuja concentração foi ajustada em câmara de Neubauer em 5,5 x $10^{6}$ esporos / $\mathrm{ml}$ de suspensão. Em cada placa de Petri de vidro de $15 \mathrm{~cm}$ de diâmetro, esterilizada, contendo $40 \mathrm{ml}$ de meio MEA + manitol solidificado, com potencial de -1,4 MPa, distribuiu-se 1,0 $\mathrm{ml}$ da suspensão de esporos do fungo sobre o meio.

A utilização do manitol no potencial de $-1,4 \mathrm{MPa}$ foi definida tendo-se como base resultados de ensaios anteriores, onde foi determinada a concentração osmótica do meio MEA ideal para o desenvolvimento do fungo e suficiente para retardar a germinação das sementes de milho, preservando a qualidade fisiológica destas. Para o desenvolvimento das colônias de $A$. strictum, deu-se a incubação das mesmas durante cinco dias, a $20^{\circ} \mathrm{C}$ e fotoperíodo de $12 \mathrm{~h}$, em BOD. As sementes de milho após desinfestadas com $\mathrm{NaClO} 1 \%$ por $2 \mathrm{~min}$, enxaguadas em água destilada esterilizada por duas vezes sucessivas e secas em câmara de fluxo laminar por $4 \mathrm{~h}$, foram dispostas sobre a colônia do fungo, em camada única dentro da placa de Petri, sendo incubadas a $20^{\circ} \mathrm{C}$, com fotoperíodo de $12 \mathrm{~h}$, em BOD nos períodos de exposição de $0,24,36,72,96$ e $120 \mathrm{~h}$.

Parte das sementes foi depositada sobre o meio MEA sem restrição hídrica com manitol. A incidência de $A$. strictum em sementes (Limonard, 1966) foi avaliada levando-se em consideração o ponto de desenvolvimento do inóculo do patógeno em relação às mesmas, sendo isso possível com a desinfestação, com $\mathrm{NaClO} 1 \%$ por 2 min de metade das sementes de cada tratamento.

\section{Delineamento estatístico}

$\mathrm{Na}$ avaliação do efeito da restrição hídrica sobre o crescimento micelial e em características morfológicas de $A$. strictum foram empregados 16 tratamentos e na avaliação do efeito do meio MEA, ajustado osmoticamente, na esporulação do fungo, nove tratamentos. Para ambas avaliações foram utilizadas cinco repetições para cada tratamento e parcela experimental constituída por uma placa de Petri. Para a avaliação do percentual de sementes com radículas protrundidas em meio MEA foram empregados nove tratamentos com quatro repetições em cada tratamento e a parcela experimental constituída por duas placas de Petri com 50 sementes. Para todos os ensaios empregou-se o delineamento inteiramente casualizado (DIC), em esquema fatorial, com uma parcela adicional. Para avaliação do percentual de incidência de $A$. strictum em sementes desinfestadas e não desinfestadas, foram usados 18 períodos de exposição das sementes, com quatro repetições e parcela experimental constituída por 25 sementes, em delineamento inteiramente casualizado (DIC). Para as análises estatísticas dos dados foi empregado o programa SAS.

\section{RESULTADOS E DISCUSSÃO}

\section{Efeito da restrição hídrica do meio MEA no desenvol- vimento de colônias de $\boldsymbol{A}$. strictum}

$\mathrm{O}$ crescimento micelial de $A$. strictum não foi reduzido por nenhum dos solutos e potenciais osmóticos avaliados (Tabela 2). Após análise de regressão não se observou efeito significativo para potenciais isoladamente ou para a interação meio de cultura x potencial osmótico. No caso do meio MEA + manitol, que estatisticamente foi superior aos demais, observou-se efeito estimulante para crescimento micelial do fungo, tendo inclusive superado o tratamento controle em $10,3 \%$, quando considerada a média dos potenciais $-0,6 \mathrm{a}-1,4 \mathrm{MPa}$. O meio $\mathrm{MEA}+\mathrm{NaCl}$ apresentou desempenho intermediário e o meio $\mathrm{MEA}+\mathrm{KCl}$ não diferiu do controle.

O estímulo do manitol ao crescimento de fungos anteriormente foi relatado por Sommers et al. (1970) para Phytophothora megasperma Drechsler. Segundo Alam et al. (1996), este estímulo provavelmente deveu-se à absorção de solutos e a um melhor ajuste osmótico das células fúngicas. Para Cryphonectria parasitica (Murrill) Barr, Gao \& Shain (1995) observaram maior crescimento em meio modificado

TABELA 1 - Quantidades de manitol, $\mathrm{KCl}$ e $\mathrm{NaCl}$ utilizadas no ajuste do meio MEA em diferentes potenciais osmóticos (MPa)

\begin{tabular}{lccc}
\hline \hline \multirow{2}{*}{ MPa } & \multicolumn{3}{c}{ Solutos adicionados ao meio MEA $\left(\mathrm{g. \mathbf {l } ^ { \mathbf { 1 } }}\right.$ de MEA) } \\
\cline { 2 - 4 } & Manitol & KCl & NaCl \\
\hline$-0,48$ (controle) & 0,0 & 0,0 & 0,0 \\
$-0,6$ & 8,9 & 2,0 & 1,5 \\
$-0,8$ & 23,5 & 5,2 & 4,1 \\
$-1,0$ & 37,9 & 8,6 & 6,7 \\
$-1,2$ & 52,0 & 11,9 & 9,3 \\
$-1,4$ & 65,8 & 15,3 & 11,9 \\
\hline
\end{tabular}

Fonte: Michel \& Radcliffe (1995) 
TABELA 2 - Taxa de crescimento micelial (T.C.M.) e características morfológicas avaliadas em colônias de $A$. strictum em meio MEA osmoticamente ajustado pela adição de manitol, $\mathrm{KCl}$ e $\mathrm{NaCl}$ em diferentes potenciais osmóticos (MPa)

\begin{tabular}{|c|c|c|c|c|c|c|}
\hline \multirow{2}{*}{ Meio } & \multirow{2}{*}{ MPa } & \multicolumn{5}{|c|}{$\begin{array}{l}\text { Características morfológicas } \\
\end{array}$} \\
\hline & & T.C.M(2) & C.M.T. & T.M. & F.M.C. & P.C. \\
\hline \multirow[t]{2}{*}{ MEA (controle) } & $-0,48$ & $2,75 \mathrm{c}^{(3)}$ & $\mathrm{A}$ & $\mathrm{F}$ & $\mathrm{R}$ & $\mathrm{B}-\mathrm{B}$ \\
\hline & $-0,6$ & $2,98 \mathrm{a}$ & $\mathrm{S}$ & $\mathrm{L}$ & $\mathrm{R}$ & Rs-Rs \\
\hline MEA & $-0,8$ & $3,05 \mathrm{a}$ & $\mathrm{S}$ & $\mathrm{L}$ & $\mathrm{R}$ & Rs-Rs \\
\hline+ & $-1,0$ & $3,08 \mathrm{a}$ & $\mathrm{S}$ & $\mathrm{L}$ & $\mathrm{R}$ & Rs-Rs \\
\hline \multirow[t]{3}{*}{ Manitol } & $-1,2$ & $3,08 \mathrm{a}$ & $\mathrm{S}$ & $\mathrm{L}$ & $\mathrm{R}$ & Rs-Rs \\
\hline & $-1,4$ & $3,14 \mathrm{a}$ & S & $\mathrm{L}$ & $\mathrm{R}$ & Rs-Rs \\
\hline & $-0,6$ & $2,93 \mathrm{~b}$ & $\mathrm{~S}$ & $\mathrm{~L}$ & $\mathrm{R}$ & Rs-Rs \\
\hline MEA & $-0,8$ & $2,99 \mathrm{~b}$ & $\mathrm{~S}$ & $\mathrm{~L}$ & $\mathrm{R}$ & Rs-Rs \\
\hline+ & $-1,0$ & $2,95 \mathrm{~b}$ & $\mathrm{~S}$ & $\mathrm{~L}$ & $\mathrm{R}$ & Rs-Rs \\
\hline \multirow[t]{3}{*}{$\mathrm{NaCl}$} & $-1,2$ & $2,96 \mathrm{~b}$ & S & $\mathrm{L}$ & $\mathrm{R}$ & Rs-Rs \\
\hline & $-1,4$ & $2,96 \mathrm{~b}$ & $\mathrm{~S}$ & $\mathrm{~L}$ & $\mathrm{R}$ & Rs-Rs \\
\hline & $-0,6$ & $2,80 \mathrm{c}$ & $\mathrm{S}$ & $\mathrm{L}$ & $\mathrm{R}$ & Rs-Rs \\
\hline MEA & $-0,8$ & $2,78 \mathrm{c}$ & $\mathrm{S}$ & $\mathrm{L}$ & $\mathrm{R}$ & Rs-Rs \\
\hline+ & $-1,0$ & $2,77 \mathrm{c}$ & $\mathrm{S}$ & $\mathrm{L}$ & $\mathrm{R}$ & Rs-Rs \\
\hline \multirow[t]{2}{*}{ KCl } & $-1,2$ & $2,77 \mathrm{c}$ & $\mathrm{S}$ & $\mathrm{L}$ & $\mathrm{R}$ & Rs-Rs \\
\hline & $-1,4$ & $2,75 \mathrm{c}$ & $\mathrm{S}$ & $\mathrm{L}$ & $\mathrm{R}$ & Rs-Rs \\
\hline
\end{tabular}

com sacarose, sendo esta considerada como fonte adicional de energia a ser metabolizada pelo patógeno.

Por meio da análise de características morfológicas (Tabela 2), pode-se verificar que em meio osmoticamente ajustado, a morfologia típica de colônias de $A$. strictum diferiu dos padrões apresentados pelo tratamento controle ($0,48 \mathrm{MPa})$, exceto para a forma regular $(\mathrm{R})$ das colônias. De modo geral, colônias do patógeno desenvolvidas em meio MEA acrescido de manitol, $\mathrm{NaCl}$ e $\mathrm{KCl}$ apresentaram crescimento micelial superficial, textura lisa, colônias regulares e pigmentação rosa salmão (10R - 7/10) (Tabela 2; Figura 1A) e o controle (MEA) apresentou crescimento micelial aéreo, textura floculosa e pigmentação bege $(2.5 \mathrm{Y}$ 8.5/2). Segundo Alam et al. (1996), a cor típica das colônias pode ser ou não influenciada pelos solutos osmóticos nas diferentes concentrações induzidas ao meio padrão de crescimento. Em Ascochyta paspali Sacc., Morley et al. (1993) observaram que a coloração das colônias não sofreu alteração em função dos solutos utilizados, e sim com o potencial hídrico adotado.

Para a esporulação de $A$. strictum in vitro, não foram observados efeitos significativos para os potenciais avaliados e para a interação (meios x potenciais) (Tabela 3). Quando comparado ao meio MEA padrão (controle), o meio MEA + manitol aumentou em $28,6 \%$ a esporulação do fungo considerando os potenciais testados. A esporulação do $A$. strictum no meio MEA + $\mathrm{NaCl}$ não diferiu estatisticamente do meio MEA padrão. Nenhum dos solutos utilizados no ajuste osmótico do meio padrão MEA afetou negativamente a indução de esporulação de $A$. strictum.
Efeito do ajuste osmótico do meio MEA na protrusão radicular de sementes de milho

Os menores percentuais de protrusão radicular em sementes de milho incubadas por até cinco dias, foram observadas em MEA + manitol (-1,4 MPa) e MEA + $\mathrm{NaCl}(-1,2 \mathrm{e}$ $-1,4 \mathrm{MPa}$ ) (Tabela 4; Figura 1B). Esses resultados assemelhamse aos valores obtidos por Carvalho (1999) para sementes de feijoeiro com o soluto manitol em meio BDA, utilizando potenciais osmóticos - 0,8 e -1,0 MPa. Segundo Costa (2000), a adição de sacarose, cloreto de potássio e manitol ao meio BSA (batata, sacarose e ágar) inibiu a germinação de sementes de feijoeiro por até $144 \mathrm{~h}$. Nesse caso, o efeito mais expressivo foi observado para KCl. Campos \& Assunção (1990), avaliaram o efeito do estresse hídrico induzido em substratos modificados com soluções osmóticas de $\mathrm{NaCl}$ e $\mathrm{Na}_{2} \mathrm{SO}_{4}$ e verificaram que o potencial $-0,8 \mathrm{MPa}$ prejudicou a germinação de sementes de arroz (Oryza sativa L.). Isto ocorreu provavelmente devido ao efeito fitotóxico do íon $\mathrm{Na}^{2+}$. No presente estudo, a situação foi semelhante para as sementes de milho, incubadas em meio $\mathrm{MEA}+\mathrm{NaCl}$, para a condição de restrição hídrica mais pronunciada $(-1,4 \mathrm{MPa})$. As sementes a ela submetidas não germinaram mesmo após oito dias da incubação, indicando que a qualidade fisiológica das mesmas foi afetada pelo condicionamento osmótico imposto ao meio de incubação.

Nível de infecção de sementes de milho, incubadas sobre colônias de $\boldsymbol{A}$. strictum desenvolvidas em meio MEA osmoticamente modificado

Os maiores percentuais de incidência de A. strictum 
Técnica de restrição hídrica: efeito sobre Acremonium strictum, protrusão...

TABELA 3 - Número de esporos produzidos por Acremonium strictum cultivado em meio de malte-agar (MEA) osmoticamente ajustado pela adição de manitol e $\mathrm{NaCl}$ em diferentes potenciais osmóticos $(\mathrm{MPa})$

\begin{tabular}{llc}
\hline \hline Meio & MPa & $\begin{array}{c}\text { Número de } \\
\text { esporos } \mathbf{~ 1 0} / \mathbf{m l}^{(1)}\end{array}$ \\
\hline MEA (controle) & $-0,48$ & $65,8 \mathrm{~b}^{(2)}$ \\
\hline MEA & $-0,8$ & $95,2 \mathrm{a}$ \\
+ & $-1,0$ & $90,2 \mathrm{a}$ \\
Manitol & $-1,2$ & $89,4 \mathrm{a}$ \\
& $-1,4$ & $93,6 \mathrm{a}$ \\
\hline \multirow{2}{*}{ MEA } & $-0,8$ & $73,8 \mathrm{~b}$ \\
+ & $-1,0$ & $69,2 \mathrm{~b}$ \\
NaCl & $-1,2$ & $74,4 \mathrm{~b}$ \\
& $-1,4$ & $77,8 \mathrm{~b}$ \\
\hline
\end{tabular}

(1) Dados originais transformados por $\sqrt{x \quad 1}$

${ }^{(2)}$ Médias seguidas pela mesma letra nas colunas não diferem estatisticamente pelo teste de Tukey $(\mathrm{P}=5 \%)$.

TABELA 4 - Percentagem de sementes de milho (Zea mays) apresentando protrusão radicular em meio de extrato de malte-agar (MEA) osmoticamente ajustado pela adição de manitol e $\mathrm{NaCl}$ em diferentes potenciais osmóticos $(\mathrm{MPa})$ e dois períodos de incubação (cinco e oito dias)

\begin{tabular}{lccc}
\hline \hline \multirow{2}{*}{ Meio } & \multirow{2}{*}{ MPa } & \multicolumn{2}{c}{ Protrusão radicular (\%) ${ }^{(1)}$} \\
\cline { 2 - 4 } & & Após 5 dias & Após 8 dias \\
\hline MEA (controle) & $-0,48$ & $46 \mathrm{a}^{(2)}$ & $80 \mathrm{a}^{(2)}$ \\
\hline \multirow{2}{*}{ MEA } & $-0,8$ & $27 \mathrm{~b}$ & $48 \mathrm{~b}$ \\
+ & $-1,0$ & $22 \mathrm{~b}$ & $38 \mathrm{~b}$ \\
Manitol & $-1,2$ & $13 \mathrm{c}$ & $27 \mathrm{~b}$ \\
& $-1,4$ & $2 \mathrm{~d}$ & $23 \mathrm{~b}$ \\
\hline \multirow{2}{*}{ MEA } & $-0,8$ & $38 \mathrm{a}$ & $69 \mathrm{~b}$ \\
+ & $-1,0$ & $22 \mathrm{~b}$ & $42 \mathrm{~b}$ \\
NaCl & $-1,2$ & $2 \mathrm{~d}$ & $20 \mathrm{c}$ \\
& $-1,4$ & $0 \mathrm{~d}$ & $0 \mathrm{~d}$ \\
\hline
\end{tabular}

${ }^{(1)}$ Dados originais transformados por $\sqrt{x \quad 1}$

${ }^{(2)}$ Médias seguidas pela mesma letra nas colunas não diferem estatisticamente pelo teste de Tukey $(\mathrm{P}=5 \%)$. foram observados para sementes de milho não desinfestadas $(38 \%)$ e desinfestadas $(36 \%)$ com $\mathrm{NaClO}$ e expostas à colônia do patógeno em meio MEA + manitol (-1,4 MPa) por $120 \mathrm{~h}$ (Figuras 1C e 2). Para os tempos de exposição avaliados, não houve diferença entre percentuais de incidência de $A$. strictum observados para sementes desinfestadas ou não. Del Giúdice (1996) observou elevada incidência de sementes de soja [Glycine $\max (\mathrm{L}$.) Merril] infetadas por microrganismos

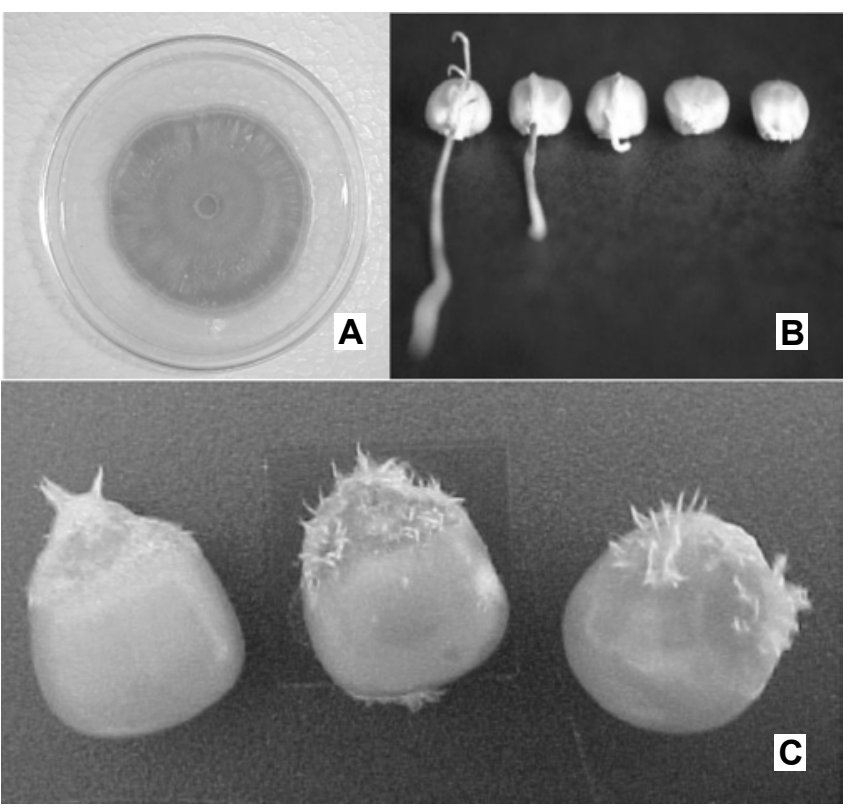

FIG. 1 - Aspecto geral da colônia de Acremonium strictum em meio de malte-agar (MEA) osmoticamente ajustado (A). Detalhe da protrusão radicular em sementes de milho (Zea mays) expostas ao meio MA + manitol em diferentes potenciais $(\mathrm{MPa})$, por até $120 \mathrm{~h}$ (B). Aspecto geral de sementes de milho inoculadas por $120 \mathrm{~h}$ com A. strictum, pela técnica de restrição hídrica em meio MEA + manitol, no potencial de -1,4 MPa (C).

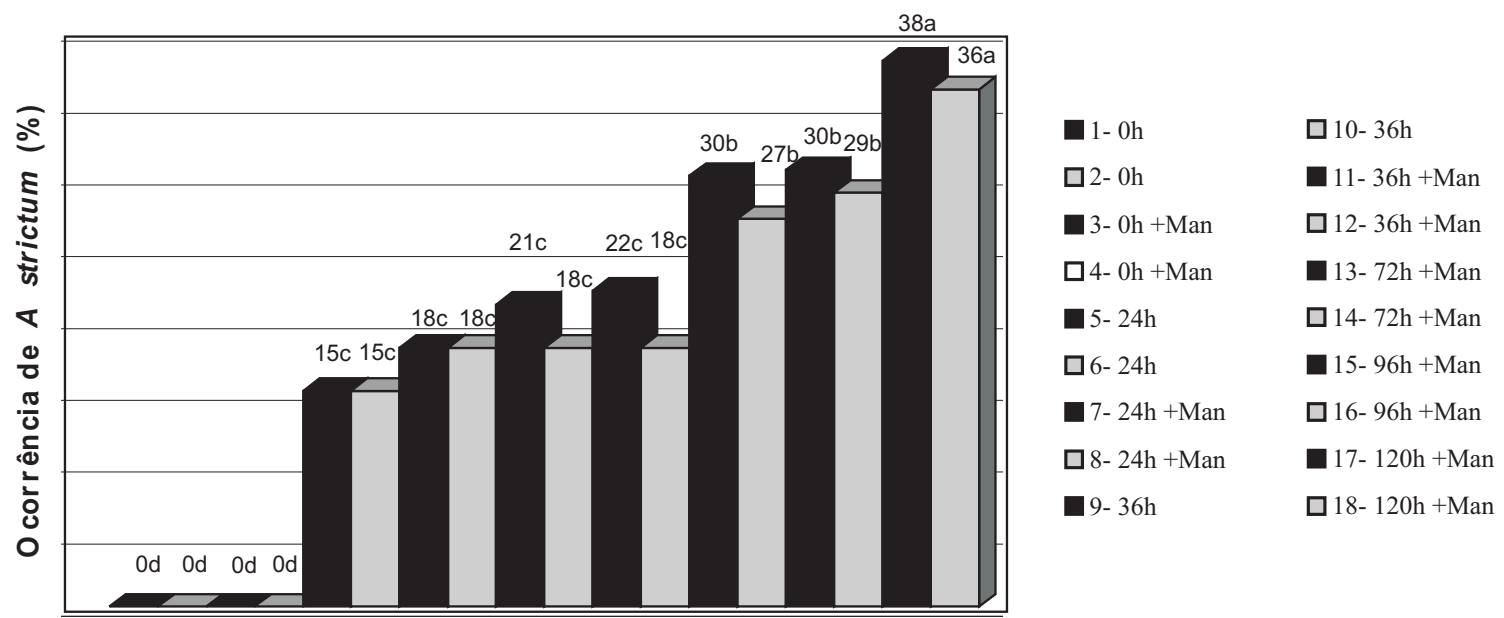

FIG. 2 - Ocorrência (\%) de Acremoniu strictum em sementes de milho (Zea mays) não desinfestadas e desinfestadas com NaClO, e expostas ao patógeno por diferentes períodos em meio extrato de malte ágar acrescido de manitol (+Man) ou não, ajustado à concentração osmótica de -1,4 MPa. Médias seguidas da mesma letra não diferem estatisticamente pelo teste de Tukey (P=5\%). 
utilizando manitol em potenciais osmóticos mais negativos, a pa1rtir do quarto dia de pré-condicionamento. Bewley \& Black (1994) citam que, na fase inicial do processo de germinação, as sementes exsudam íons e constituintes orgânicos, os quais podem estimular o desenvolvimento de microrganismos. No presente estudo, sementes inoculadas por 24 e $36 \mathrm{~h}$, e por 72 e $96 \mathrm{~h}$, respectivamente, não diferiram quanto à incidência de $A$. strictum. Foi observado também que $A$. strictum mostrou-se pouco agressivo às sementes de milho, pois os dados de incidência em sementes não desinfestadas com $\mathrm{NaClO}$ e expostas à colônia do fungo por até $120 \mathrm{~h}$ podem ser considerados relativamente baixos. Entretanto, a incidência do fungo foi proporcionalmente superior em sementes desinfestadas, indicando que $A$. strictum se estabeleceu mais internamente aos tecidos da semente nos maiores períodos de inoculação. As análises sanitárias de sementes de milho realizadas rotineiramente nos laboratórios de Patologia de Sementes do Brasil têm revelado que os percentuais de ocorrência natural em geral dificilmente ultrapassam 30\% (J. C. Machado, comunicação pessoal).

\section{REFERÊNCIAS BIBLIOGRÁFICAS}

ALAM, S., JOYCE, D. \& WEARING, A. Effects of equilibrium relative humidity on in vitro growth of Botrytis cinerea and Alternaria alternata. Australian Journal of Experimental Agriculture 36:383-388. 1996.

BEWLEY, J.D. \& BLACK, M. Seeds: physiology of development and germination. $2^{\text {nd }}$ ed. New York. Plenum Press. 1994.

CAMPOS, I.S. \& ASSUNÇÃO, M.V. Estresse salino e hídrico na germinação e vigor do arroz. Pesquisa Agropecuária Brasileira 25:857-862. 1990.

CARVALHO, J.C.B de. Uso da restrição hídrica na inoculação de Colletotrichum lindemuthianum em sementes de feijoeiro (Phaseolus vulgaris L.). (Dissertação de Mestrado). Lavras. Universidade Federal de Lavras. 1999.

CHAVES, K.C., RAVA, C.A. \& COSTA, J.L.S. Inoculação de sementes e controle químico da sarna do feijoeiro comum (Colletotrichum sp.). Resumos expandidos, $6^{\text {a }}$ Reunião Nacional de Feijão, Salvador, BA. 1999. pp.223-225.

COSTA, M.L.N. Inoculação de Fusarium oxysporum f.sp. phaseoli por meio da restrição hídrica. (Dissertação de Mestrado). Lavras. Universidade Federal de Lavras. 2000.

DEL GIÚDICE, M.P. Condicionamento osmótico de sementes de soja (Glycine max (L.) Merril). (Tese de Doutorado). Viçosa. Universidade Federal de Viçosa. 1996.

GAO, S. \& SHAIN, L. Effect of osmotic potential on virulent and hypovirulent strains of the chesnut blight fungus. Canadian Journal of Forest Research 25:1024-1029. 1995.

HEYDECKER, W., HIGGINS, J. \& GULLIVER, R.L. Accelerated germination by osmotic seed treatment. Nature 246:42-44. 1973.

HEYDECKER, W., HIGGINS, J. \& TURNER, Y.J. Invigoration of seeds? Seed Science \& Technology 3:881-888. 1975.
LIMONARD, T. A modified blotter test for seed health. Netherlands Journal of Plant Pathology 72:319-321. 1966.

MICHEL, B.E. \& RADCLIFFE, D. A computer program relating solute potential to solution composition for five solutes. Agronomy Journal 87:126-130. 1995.

MORLEY, T.B., WILLIAMS, B.L. \& PRICE, T.V. The effects of water stress on the incidence and severity of paspalum leaf blight and on Ascochyta paspali. Australian Plant Pathology 22:105-110. 1993.

MUNSELL, A.H. Munsell book of color: glossy finish collection (2.5R-10G). Maryland. Macbeth Division. 1976.

PERES, A.P. Detecção de Sclerotinia sclerotiorum (Lib.) de Bary em sementes de feijoeiro (Phaseolus vulgaris L.) e soja (Glycine $\max ($ L.) Merri): desenvolvimento de metodologias. (Dissertação de Mestrado). Lavras. Universidade Federal de Lavras. 1996.

PILL, W.G. Low water potential and pressing germination treatments to improve seed quality. In: Barsa, A.S. (Ed.). Seed quality: basic mechanisms and agricultural implications. New York. Food Products Press. 1994. pp.319-359.

RAVA, A.C. \& SARTORATO, A. Eficiência de fungicidas no controle de Colletotrichum lindemuthianum inoculado em sementes de feijoeiro. Anais, $5^{\text {a }}$ Reunião Nacional de Pesquisa de Feijoeiro, Goiânia, GO. 1996. pp.210-212.

RICHARDSON, M.J. An annotated list of seed borne diseases. $3^{\text {nd }}$ ed. Kew Surrey. ISTA. 1980.

SHURTLEFF, M.C. Compendium of maize diseases. St. Paul. American Phytopathological Society. 1992.

SMITH, B.M. \& WHITE, D.G. Diseases of maize. In: Sprague, G.F. $\&$ Dudley, Y.W. (Eds.). Maize and maize improvement. $3^{\text {nd }}$ ed. Madison. Agronomy monograph, 18. 1988. pp.687-766.

SMITH, D. \& ONIONS, A.H.S. The preservation and maintenance of living fungi. Kew. Commonwealth Mycological Institute. 1994.

SOMMERS, L.E., HARRIS, R.F., DALTON, F.N. \& GARDNER, W.R. Water potential relations of three root-infecting Phytophthora species. Phytopathology 60:932-934. 1970.

TANAKA, M.A.S. Patogenicidade e transmissão por sementes do agente causal da ramulose do algodeiro. (Tese de Doutorado). Piracicaba. Escola Superior de Agricultura Luiz de Queiroz. 1990.

TANAKA, M.A.S. \& CORRÊA, M.U. Influência de Aspergillus e Penicillium no armazenamento de sementes de feijoeiro (Phaseolus vulgaris L.). Fitopatologia Brasileira 6:451-456. 1981.

TEIXEIRA, H., VIEIRA, M.G.G.C. \& MACHADO, J.C. Avaliação dos efeitos do tratamento químico e biológico de Colletotrichum gossypii South. em sementes de algodoeiro (Gossypium hirsutum L.). Ciência e Agrotecnologia 21:413-418. 1997a.

TEIXEIRA, H., VIEIRA, M.G.G.C. \& MACHADO, J.C. Influência de Colletotrichum gossypii South. no desenvolvimento inicial do algodão (Gossypium hirsutum L.) em função da localização do inóculo e desinfestação das sementes. Revista Brasileira de Sementes 19;9-13. 1997b.

VIEIRA, M.G.G.C. Utilização de marcadores moleculares no monitoramenteo do nível de deterioração e da qualidade sanitária de sementes do algodoeiro. (Tese de Doutorado). Lavras. Universidade Federal de Lavras. 1996. 